\title{
Analyzing the Downside Risk of Exchange-Traded Funds: Do the Volatility Estimators Matter?
}

\author{
Jying-Nan Wang ${ }^{1}$, Lu-Jui Chen ${ }^{2}$, Hung-Chun $\mathrm{Liu}^{3} \&$ Yuan-Teng Hsu ${ }^{4}$ \\ ${ }^{1}$ Department of Applied Economics, Fo Guang University, Taiwan \\ ${ }^{2}$ Department of International Business, Ming Chuan University, Taiwan \\ ${ }^{3}$ Department of Finance, Minghsin University of Science \& Technology, Taiwan \\ ${ }^{4}$ College of Management, Yuan Ze University, Taiwan
}

Correspondence: Hung-Chun Liu, Department of Finance, Minghsin University of Science \& Technology, No. 1, Xinxing Rd., Xinfeng Hsinchu 30401, Taiwan (R.O.C.). Tel: 886-3-559-3142. E-mail: hungchun65@gmail.com

Received: September 20, 2015

Accepted: October 8, 2015

Online Published: December 25, 2015

doi:10.5539/ijef.v8n1p1

URL: http://dx.doi.org/10.5539/ijef.v8n1p1

\begin{abstract}
This paper aims to propose the augmented GJR-GARCH (GJR-GARCH ${ }_{M}$ ) model that extends the GJR-GARCH model by comprising overnight returns volatility (ONV), daily high-low prices range (PK), and fear index (VIX) as explanatory variables for the GJR's variance equation, respectively. The proposed models are used to estimate the daily value-at-risk values and evaluate their downside risk management performance for the SPDRs covering the period from 2009 to 2014. Empirical results show that the GJR-GARCH $\mathrm{H}_{\mathrm{M}}$ model outperforms the GJR-GARCH model for most cases, suggesting that the GJR-GARCH-based VaR forecasts can be moderately improved with the additional information embodied in the ONV, PK and VIX volatility estimators. In addition, daily high-low prices range and VIX are far more informative than the overnight volatility estimator for improving the GJR-GARCH-based VaR forecasts. Risk managers can employ the proposed models for estimating and controling the potential loss of ETFs in the face of financial catastrophes.
\end{abstract}

Keywords: exchange-traded funds, SPDRs, value-at-risk, volatility estimator, GJR

\section{Introduction}

Exchange-traded funds (ETFs) are very prevalent and have become popularly adopted investment tools among common investors over recent years. ETFs are attractive as investments because they have many advantages, such as low fee ratios, tax efficiency, diversified-portfolio and stock-like characteristics. The American Stock Exchange introduces the Standard and Poor's Depositary Receipts (SPDRs, or called Spider) in the early 1990s, which are backed by an equity portfolio that closely traces the S\&P 500 index. By far, the Spider is the most actively traded and the largest passive ETF worldwide, with US\$215.91 billion market value as at January, 2015.

Researchers have long been observed that volatility of financial assets returns are often described by several stylized facts, such as time-varying, clustering, and persistence features.The ARCH (autoregressive conditional heteroskedastic, ARCH) model proposed by Engle (1982) and the GARCH (generalized autoregressive conditional heteroskedastic, GARCH) model advocated by Bollerslev (1986) respond to deal with these stylized phenomena. Since then, volatility forecasting technique has been dominated by a variety of the GARCH genre of models, especially for the asymmetric GARCH model. Glosten et al. (1993) propose the so-called GJR-GARCH model which is a simple class of GARCH-type models which can capture leverage effects of positve news and negative news on conditional volatility.

To the best of our knowledge, a large volume of recent studies have been investigated and written about the value-at-risk (VaR) issue for various financial markets by using GARCH techniques, such as Angelidis et al. (2004), Huang and Lin (2004), Liu and Hung (2010), Orhan and Köksal (2012) and So and Yu (2006) for stock markets, Al Janabi (2006), Bams et al. (2005) and So and Yu (2006) for foreign exchange rate markets, Chan and Gray (2006), Sadeghi and Shavvalpour (2006) and Sadorsky (2006) for energy markets. However, despite the importance of VaR on financial risk management and the popularity of ETFs for common investors, there seems to have been relatively little work endeavored on ETFs. 
Brooks et al. (2000) advocate the overnight return volatility (ONV) in order to capture accumulated overnight information that would be beneficial for capturing the persistence in the conditional heteroscedasticity of stock returns. Motivated by the daily price range, on the one hand, Parkinson (1980) exploits the scaled high-low price ranges to construct the daily PK estimator based on the assumption which intraday trading prices follow a random walk process. On the other hand, Garman and Klass (1980) propose the so-called GK estimator by including opening and closing trading prices in addition to price range, with similar assumptions to the PK. In addition, Rogers and Satchell (1991) develop the RS volatility estimator by considering the drift in the price process. In 1993, Chicago Board Options Exchange develops the fear index (VIX, or called implied volatility) that is acquired from the S\&P 500 index option prices data via an option pricing model.

In recent years, the wide availability of intraday data has encouraged researchers to explore their information value in modeling and predicting the volatility of financial assets, such as Blair et al. (2001), Corrado and Truong (2007), Fuertes et al. (2009), Koopman et al. (2005), Vipul and Jacob (2007) and the reference therein. However, despite a large volume of existing literature on volatility forecasting, none of them investigates the prices information which is embodied in the ONV, PK and VIX volatility estimators for improving predictive accuracy of daily value-at-risk forecasts in ETF. Thus, this paper aims to propose the augmented GJR model that extends the traditional GJR-GARCH model by comprising three volatility estimators, overnight volatility (ONV), daily prices range (PK), and fear index (VIX) as explanatory variables for the variance equations in GJR model. The proposed models are used to estimate their daily $\mathrm{VaR}$ values and evaluate their downside risk management performance for the SPDRs returns spanning from 2009 to 2014.

The remainder of this paper is organized as follows. The data and econometric methodology are provided in Section 2, followed in Section 3 by the empirical results of daily VaR forecasts performance for SPDRs across alternative confidence levels. The final Section summarizes the conclusions drawn from this paper.

\section{Methodology}

\subsection{Data and Descriptive Analysis}

The data analyzed in this paper comprises of the daily open, high, low, and closing prices data on SPDRs as well as the VIX data obtained from the Yahoo Finance website. The sample period for these daily data covers from 2 January 2009 to 31 December 2014 for a total of 1,510 trading days. The first four years (1,006 observations) are used as the in-sample period for estimation purpose, while the remaining two years (504 observations) are left for out-of-sample forecast evaluation.

Table 1. Descriptive statistics of daily returns for the SPDRs

\begin{tabular}{cccccccc}
\hline Mean $(\%)$ & Std. & Min & Max & Skew & Kurt & J-B & Q $_{s}(12)$ \\
\hline 0.053 & 1.142 & -6.734 & 6.960 & $-0.270^{*}$ & $4.484^{*}$ & $1283.019^{*}$ & $796.501^{*}$ \\
\hline
\end{tabular}

Note. This table presents the descriptive statistics of daily returns for the Standard \& Poor's Depositary Receipts. J-B represents the statistics of Jarque and Bera (1987)'s normal distribution test. $Q_{s}(12)$ refers to the Ljung-Box Q test statistic of the squared return series for up to the 12th order serial correlation. * indicates significance at the $1 \%$ level.

Table 1 provides the descriptive statistics of the daily returns for the Standard \& Poor's Depositary Receipts. As showed in Table 1 , the daily average return of SPDR is $0.053 \%$, and very closes to zero. The skewness and kurtosis of returns series exhibit significant evidence, meaning that the returns have a left skewness, and the distribution of the returns is much fat-tailed and high-peaked than normal distribution. The Jarque and Bera (1987) test statistic also shows that the daily SPDR returns are not normal-distributed. Moreover, the $\mathrm{Q}_{\mathrm{s}}(12)$ test statistic exhibits linear dependence for the squared returns and exists siginificant $\mathrm{ARCH}$ effects.

\subsection{Augmented GJR Model}

We propose the augmented GJR model which extends the GJR-GARCH model of Glosten et al. (1993) by including various volatility estimators (ONV, PK and VIX), respectively, for its variance equation as follows:

$$
\begin{aligned}
& R_{t}=\mu+\varepsilon_{t}, \quad \varepsilon_{t}=\sigma_{t} z_{t},\left.\quad z_{t}\right|_{\Omega_{t-1}} \sim N I D(0,1) \\
& \sigma_{t}^{2}=\omega+\alpha \varepsilon_{t-1}^{2}+\gamma d_{t-1} \varepsilon_{t-1}^{2}+\beta \sigma_{t-1}^{2}+\delta v_{t-1}
\end{aligned}
$$


where $R_{t}$ is daily SPDRs return; $\mu$ is the conditional mean of returns; $\varepsilon_{t}$ represents the innovation process; $z_{t}$ denotes the standardized residual with zero mean and unit variance; $\sigma_{t}^{2}$ is the conditional variance. $d_{t-1}$ denotes the indicator function that takes the value of unity if $\varepsilon_{t-1}<0$, and 0 otherwise. The indicator variable differentiates between good news and bad news impacts, so that leverage effects are captured by $\gamma$. Thus, in the augmented GJR model, good news has an impact of $\alpha$, and bad news has an impact of $(\alpha+\gamma)$, with bad (good) news having a greater shock on volatility if $\gamma>0 \quad(\gamma<0)$. Finally, $v_{t-1}$ denotes a volatility estimator made at day $t-1$, including ONV (overnight volatility), PK (daily high-low price range), and VIX (fear index). Table 2 provides a synopsis of these volatility estimators:

Table 2. The synopsis of various volatility estimators

\begin{tabular}{|c|c|c|c|}
\hline Abbreviation of volatility estimators & Studies & Formula or explanation & \\
\hline$O N V$ & Brooks et al. (2000) & $\hat{\sigma}_{O N V, t}^{2}=\left(\ln \left(O_{t} / C_{t-1}\right)\right)^{2}$ & (3) \\
\hline$P K$ & Parkinson (1980) & $\hat{\sigma}_{\mathrm{PK}, \mathrm{t}}^{2}=(4 \ln 2)^{-1} \cdot\left(\ln \left(\mathrm{H}_{\mathrm{t}} / \mathrm{L}_{\mathrm{t}}\right)\right)^{2}$ & (4) \\
\hline$V I X$ & - & $\begin{array}{l}\text { VIX is a popular measure of the fear index of S\&P } 500 \\
\text { index options, which represents one measure of the } \\
\text { market's expectation of stock market volatility over the } \\
\text { next } 30 \text { day period. For consistent scaling with ONV } \\
\text { and PK, the VIXs are squared and divided by } 252 \text {. }\end{array}$ & \\
\hline
\end{tabular}

Note. This table presents the various volatility estimators employed in this paper. $O_{t}, H_{t}, L_{t}$ and $C_{t}$ denote the opening, high, low, and closing prices at day $t$, respectively.

\subsection{Downside Risk Measurement and Performance Evaluation}

The GJR-based VaR forecasts for a one-day holding period can be calculated as follows:

$$
\operatorname{VaR}_{t}=Z_{\alpha_{1}} \cdot \hat{\sigma}_{t}+\mu
$$

Where $Z_{a_{1}}$ denotes the corresponding quantile of the standard normal distribution at $a_{1}$, while $\hat{\sigma}_{t}$ is the volatility forecast generated from either GJR, GJR-ONV, GJR-PK or GJR-VIX model.

In order to backtesting, this paper uses a likelihood-ratio test of Kupiec (1995) to examine whether the true failure rate is statistically consistent with theoretical failure rate of the VaR model. The null hypothesis of the failure rate $P$ is tested against the alternative hypothesis that the failure rate is different from $P$. The $\operatorname{LR}_{\mathrm{uc}}$ statistic can be formulated as follows:

$$
L R_{u c}=2 \ln \left[\frac{\hat{\pi}^{n_{1}}(1-\hat{\pi})^{n_{0}}}{P(1-P)^{n_{0}}}\right] \sim \chi^{2}(1)
$$

where $\hat{\pi}=n_{1} /\left(n_{0}+n_{1}\right)$ denotes the maximum likelihood estimate of $P$, and $n_{l}$ is a Bernoulli random variable that represents the total number of VaR violations. (Note 1)

Christoffersen (1998) constructs the sophisticated conditional coverage test $\left(\mathrm{LR}_{\mathrm{cc}}\right)$ which jointly examines whether the total number of violations is equal to the expected one, and the VaR violations are independently distributed. Given the realizations of the SPDRs returns series $R_{t}$ and the set of VaR estimates, the indicator variable $I_{t}$ can be formulated as follows:

$$
I_{t}= \begin{cases}1 & \text {,if } R_{t}<V a R_{t} \\ 0 & \text {,if } R_{t} \geq V a R_{t}\end{cases}
$$

Since accurate VaR estimates exhibit the property of correct conditional coverage, the $I_{t}$ series must display both correct unconditional coverage and serial independence. The $\mathrm{LR}_{\mathrm{cc}}$ test is a joint test of these two properties, and the related test statistic is $\mathrm{LR}_{\mathrm{cc}}=\mathrm{LR}_{\mathrm{uc}}+\mathrm{LR}_{\mathrm{ind}}$ as we condition on the first observation. Thus, under the null hypothesis that the failure process is independent and the expected proportion of violations equals $P$, the corresponding likelihood ratio can be formulated as follows: 


$$
\operatorname{LR}_{\mathrm{cc}}=-2 \ln \frac{(1-\mathrm{P})^{\mathrm{n}_{0}} \mathrm{P}^{\mathrm{n}_{1}}}{\left(1-\hat{\pi}_{01}\right)^{\mathrm{n}_{00}} \hat{\pi}_{01}^{\mathrm{n}_{01}}\left(1-\hat{\pi}_{11}\right)^{\mathrm{n}_{10}} \hat{\pi}_{11}^{\mathrm{n}_{11}}} \sim \chi^{2}(2)
$$

where $n_{i, j}=$ the number of observations with value $i$ followed by value $j(i, j=0,1)$, $\pi_{i j}=P\left\{I_{t}=j \mid I_{t-1}=i\right\}(i, j=0,1), \quad \hat{\pi}_{01}=n_{01} /\left(n_{00}+n_{01}\right), \quad \hat{\pi}_{11}=n_{11} /\left(n_{10}+n_{11}\right)$.

\section{Empirical Results and Analysis}

Table 3 presents out-of-sample daily VaR forecasts performance across the various models by reporting mean VaR, violation, failure prob., $\mathrm{LR}_{\mathrm{uc}}$ and $\mathrm{LR}_{\mathrm{cc}}$ statistics, under $90 \%, 95 \%$ and $99 \%$ confidence levels.

As shown in Table 3, the GJR model generates the highest average absolute VaR estimates at every confidence level, and followed by the GJR-ONV, GJR-PK and GJR-VIX models. Thus, the GJR and the GJR-VIX models generate the lowest and highest numbers of VaR violations, respectively.

Panel A of Table 3 provides daily VaR forecasts results for SPDRs at the $90 \%$ confidence level. We observe that either the GJR or the GJR-ONV model fails to pass the unconditional coverage test $\left(\mathrm{LR}_{\mathrm{uc}}\right)$, indicating that both traditional GJR and GJR-ONV models tend to over-predict VaR values for SPDRs returns. Moreover, the GJR model has been rejected by the conditional coverage test $\left(\mathrm{LR}_{\mathrm{cc}}\right)$, indicating that clustered violations were generated. That is, the GJR model is very slow at updating the VaR value when market volatility changes rapidly. By contrast, both the GJR-PK and the GJR-VIX models pass the coverage tests, suggesting that the empirical failure probability is statistically consistent with the prescribed one for each of them, especially for the latter model. Meanwhile, with any sudden change in market volatility, the GJR-PK and the GJR-VIX models are beneficial for rapidly updating the VaR value. Thus, the trading prices information which is implied in PK and VIX volatility measures is crucial for producing adequate daily VaR forecasts for SPDRs returns at the $90 \%$ confidence level.

For the case of $95 \%$ confidence level, we observe that the $\mathrm{LR}_{\mathrm{uc}}$ test statistic is insignificant for the GJR, GJR-ONV and GJR-PK models, indicating that the sample point estimate is statistically consistent with the prescribed confidence level of these three VaR models. The $\mathrm{LR}_{\mathrm{cc}}$ statistic further shows that the aforesaid three models also can pass the conditional coverage test, indicating that these models' performance is quite stable over time during the out-of-sample forecasting period 2013 2014. However, the GJR-VIX model fails to offer adequate $\mathrm{VaR}$ forecasts according to the $\mathrm{LR}_{\mathrm{uc}}$ test statistic.

The VaR forecasts results at the $99 \%$ confidence level are very similar to those obtained at the $95 \%$ confidence level. That is, the $\mathrm{LR}_{\mathrm{uc}}$ and $\mathrm{LR}_{\mathrm{cc}}$ statistics reported in Panel $\mathrm{C}$ of Table 3 are all insignificant, except for the GJR-VIX model, indicating that the GJR, GJR-ONV and GJR-PK models are able to produce adequate Daily VaR forecasts for SPDRs returns.

Table 3. Daily VaR forecasts results

\begin{tabular}{|c|c|c|c|c|c|}
\hline Model & Mean VaR & Violation & Failure prob. & $\mathrm{LR}_{\mathrm{uc}}$ & $\mathrm{LR}_{\mathrm{cc}}$ \\
\hline \multicolumn{6}{|c|}{ Panel A: 90\% Confidence Level } \\
\hline GJR & -1.0192 & 36 & $7.14 \%$ & 5.02 & 5.15 \\
\hline GJR-ONV & -0.9864 & 37 & $7.34 \%$ & 4.32 & 4.51 \\
\hline GJR-PK & -0.9142 & 42 & $8.33 \%$ & 1.63 & 2.52 \\
\hline GJR-VIX & -0.8268 & 53 & $10.51 \%$ & 0.14 & 0.63 \\
\hline \multicolumn{6}{|c|}{ Panel B: 95\% Confidence Level } \\
\hline GJR & -1.3166 & 25 & $4.96 \%$ & 0.00 & 0.05 \\
\hline GJR-ONV & -1.2744 & 26 & $5.15 \%$ & 0.02 & 0.10 \\
\hline GJR-PK & -1.1838 & 26 & $5.15 \%$ & 0.02 & 0.19 \\
\hline GJR-VIX & -1.0701 & 34 & $6.74 \%$ & 2.92 & 2.95 \\
\hline \multicolumn{6}{|c|}{ Panel C: 99\% Confidence Level } \\
\hline GJR & -1.8745 & 5 & $0.99 \%$ & 0.00 & 3.73 \\
\hline GJR-ONV & -1.8146 & 5 & $0.99 \%$ & 0.00 & 3.73 \\
\hline GJR-PK & -1.6897 & 7 & $1.38 \%$ & 0.68 & 3.27 \\
\hline GJR-VIX & -1.5265 & 11 & $2.18 \%$ & 5.32 & 6.48 \\
\hline
\end{tabular}

Note. This table presents daily VaR forecasts results for SPDRs at three confidence levels. The critical values of the $\mathrm{LR}_{\mathrm{uc}}$ and $\mathrm{LR}_{\mathrm{cc}}$ statistics at the $10 \%$ significance level are 2.71 and 4.61, respectively. Figures in bold text indicate rejection of the null hypothesis of correct VaR estimates at the $10 \%$ significance level. 


\section{Conclusions}

This paper proposes the augmented GJR-GARCH model that extends the GJR-GARCH model of Glosten et al. (1993) by respectively comprising overnight volatility, daily high-low prices range, and fear index as explanatory variables for the GJR-GARCH's variance equation. These VaR models are used to estimate their daily VaR values and evaluate their downside risk management performance for the SPDRs returns under 90\%, 95\% and 99\% confidence levels. Empirical results show that the augmented GJR model outperforms the GJR model for most cases, suggesting that the GJR-GARCH-based VaR forecasts can be moderately improved with the additional information embodied in the ONV, PK and VIX volatility estimators. In addition, daily high-low prices range and fear index are far more informative than the overnight volatility for improving the GJR-GARCH-based VaR forecasts. Risk managers can employ the proposed VaR models for estimating and controling the potential loss of ETFs in the face of financial catastrophes.

\section{References}

Al Janabi, M. A. M. (2006). Foreign-exchange trading risk management with value at risk: Case analysis of the Moroccan market. Journal of Risk Finance, 7(3), 273-291. http://dx.doi.org/10.1108/15265940610664951

Ana-Maria, F., Izzeldin, M., \& Kalotychou, E. (2009). On forecasting daily stock volatility: The role of intraday information and market conditions. International Journal of Forecasting, 25(2), 259-281. http://dx.doi.org/10.1016/j.ijforecast.2009.01.006

Angelidis, T., Benosa, A., \& Degiannakis, S. (2004). The use of GARCH models in VaR estimation. Statistical Methodology, 1, 105-128. http://dx.doi.org/10.1016/j.stamet.2004.08.004

Bams, D., Lehnert, T., \& Wolff, C. (2005). An evaluation framework for alternative VaR-models. Journal of International Money and Finance, 24, 944-958. http://dx.doi.org/10.1016/j.jimonfin.2005.05.004

Blair, B. J., Poon, S. H., \& Taylor, S. J. (2001). Forecasting S\&P 100 volatility: The incremental information content of implied volatilities and high frequency returns. Journal of Econometrics, 105, 5-26. http://dx.doi.org/10.1016/S0304-4076(01)00068-9

Bollerslev, T. (1986). Generalized autoregressive conditional heteroskedasticity. Journal of Econometrics, 31(3), 307-327. http://dx.doi.org/10.1016/0304-4076(86)90063-1

Brooks, C., Clare, A. D., \& Persand, G. (2000). A word of caution on calculating market-based minimum capital risk requirements. Journal of Banking and Finance, 24, 1557-1574. http://dx.doi.org/10.1016/S0378-4266(99)00092-8

Chan, K. F., \& Gray, P. (2006). Using extreme value theory to measure value-at-risk for daily electricity spot prices. International Journal of Forecasting, 22, 283-300. http://doi:10.1016/j.ijforecast.2005.10.002

Christoffersen, P. F. (1998). Evaluating interval forecasts. International Economic Review, 39, 841-862. http://dx.doi.org/10.2307/2527341

Corrado, C., \& Truong, C. (2007). Forecasting stock index volatility: Comparing implied volatility and the intraday high-low price range. Journal of Financial Research, 30(2), 201-215. http://dx.doi.org/10.1111/j.1475-6803.2007.00210.x

Engle, R. F. (1982). Autoregressive conditional heteroscedasticity with estimates of the variance of United Kindom inflation. Econometrica, 50(4), 987-1008. http://dx.doi.org/10.2307/1912773

Garman, M., \& Klass, M. (1980). On the estimation of security price volatilities from historical data. Journal of Business, 53(1), 67-78. http://dx.doi.org/10.1086/296072

Glosten, L., Jagannathan, R., \& Runkle, D. (1993). On the relation between the expected value and the volatility nominal excess return on stocks. Journal of Finance, 46, 1779-1801. http://dx.doi.org/10.1111/j.1540-6261.1993.tb05128.x

Huang, Y. C., \& Lin, B. J. (2004). Value-at-Risk analysis for Taiwan stock index futures: Fat tails and conditional asymmetries in return innovations. Review of Quantitative Finance and Accounting, 22, 79-95. http://dx.doi.org/10.1023/B:REQU.0000015851.78720.a9

Jarque, C. M., \& Bera, A. K. (1987). A test for normality of observations and regression residuals. International Statistics Review, 55, 163-172. http://dx.doi.org/10.2307/1403192

Koopman, S., Jungbacker, B., \& Hol, E. (2005). Forecasting daily variability of the S\&P 100 stock index using historical, realised and implied volatility measurements. Journal of Empirical Finance, 12(3), 445-475. 
http://dx.doi.org/10.1016/j.jempfin.2004.04.009

Kupiec, P. (1995). Techniques for verifying the accuracy of risk measurement models. Journal of Derivatives, 3, 73-84. http://dx.doi.org/10.3905/jod.1995.407942

Liu, H. C., \& Hung, J. C. (2010). Forecasting S\&P-100 stock index volatility: The role of volatility asymmetry and distributional assumption in GARCH models. Expert Systems with Applications, 37(7), 4928-4934. http://dx.doi.org/10.1016/j.eswa.2009.12.022

Orhan, M., \& Köksal, B. (2012). A comparison of GARCH models for VaR estimation. Expert Systems with Applications, 39(3), 3582-3592. http://dx.doi.org/10.1016/j.eswa.2011.09.048

Parkinson, M. (1980). The extreme value method for estimating the variance of the rate of return. Journal of Business, 53(1), 61-65. http://dx.doi.org/10.1086/296071

Rogers, L. C. G., \& Satchell, S. E. (1991). Estimating variance from high, low and closing prices. Annals of Applied Probability, 1(4), 504-512. http://dx.doi.org/10.1214/aoap/1177005835

Sadeghi, M., \& Shavvalpour, S. (2006). Energy risk management and Value at Risk modeling. Energy Policy, 34, 3367-3373. http://dx.doi.org/10.1016/j.enpol.2005.07.004

Sadorsky, P. (2006). Modeling and forecasting petroleum futures volatility. Energy Economics, 28, 467-488. http://dx.doi.org/10.1016/j.eneco.2006.04.005

So, M. K. P., \& Yu, P. L. H. (2006). Empirical analysis of GARCH models in Value at Risk estimation. Journal of International Financial Markets, Institutions \& Money, 16, 180-197. http://dx.doi.org/10.1016/j.intfin.2005.02.001

Vipul, \& Jacob, J. (2007). Forecasting performance of extreme-value volatility estimators. Journal of Futures Markets, 27(11), 1085-1105. http://dx.doi.org/10.1002/fut.20283

\section{Note}

Note 1. If the forecasted VaR can not cover the realized dollar loss, this is defined as a violation.

\section{Copyrights}

Copyright for this article is retained by the author(s), with first publication rights granted to the journal.

This is an open-access article distributed under the terms and conditions of the Creative Commons Attribution license (http://creativecommons.org/licenses/by/3.0/). 